GAPP, número 24, noviembre de 2020

Sección: ESTUDIOS

Recibido: 15-06-2020

Modificado: 08-10-2020

Aceptado: $15-10-2020$

DOI: https://doi.org/10.24965/gapp.i24.10810

Páginas: 106-119

\title{
Incidencia de las políticas globales en las legislaciones nacionales: el caso de la Convención para la Eliminación de Todas las Formas de Discriminación contra las Mujeres (CEDAW) en España
}

\author{
Impact of global policies on national legislation: the case of the \\ Convention on the Elimination of All Forms of Discrimination \\ Against Women (CEDAW) in Spain
}

\author{
Lydia Candelaria González Orta \\ Fundación Española para la Ciencia y la Tecnología (España) \\ lydia.glez.orta@gmail.com
}

\section{NOTA BIOGRÁFICA}

Doctora en Ciencias Sociales (UV) y Máster Erasmus Mundus en Estudios de Género (UniOvi y CEU). Sus intereses de investigación se centran en políticas de igualdad en organizaciones internacionales e instituciones de ciencia, derechos humanos de las mujeres y movimiento feminista transnacional. Ha colaborado en el diseño de políticas de igualdad en diferentes instituciones del sistema español de I+D+i. Actualmente está involucrada en proyectos europeos de género y ciencia en el Departamento de Ciencia Internacional de FECYT.

\section{RESUMEN}

Este artículo aborda el impacto que ha tenido la Convención de derechos humanos de las mujeres, CEDAW, en las políticas de igualdad de género en España en un momento de crisis del sistema de Derechos Humanos de Naciones Unidas. Para ello, se analiza tanto el contenido de las recomendaciones realizadas a España por el Comité CEDAW desde los ochenta como el recorrido, discurso y estrategias de los principales actores involucrados en la evaluación del Estado ante la CEDAW. El método empleado combina el análisis documental y entrevistas a informantes clave. Se sostiene que si bien la CEDAW ha servido de inspiración y legitimación internacional de las leyes de igualdad, y contribuido a la proyección internacional del movimiento feminista, la mayor complejidad y burocratización de la revisión periódica no se ha traducido en una implementación eficaz de las recomendaciones del Comité. El artículo finaliza con una discusión sobre las principales lagunas en la Administración española que obstaculizan la eficacia de la Convención en el avance de los derechos de las mujeres.

\section{PALABRAS CLAVE}

CEDAW; derechos humanos de las mujeres; políticas de igualdad; movimiento feminista.

\begin{abstract}
This article addresses the impact of CEDAW as International Treaty on women's human rights on gender equality policies in Spain. This assessment comes at a time of crisis in the UN system of Human Rights. The article analyses both the content of the CEDAW recommendations to Spain since the eighties and the path, discourse and strategies of the main actors involved in the periodic review: State Party, experts of the Committee and feminist and human rights organizations. The research method combines document analysis and interviews with key informants representing the three main actors in this process. It will be argued that
\end{abstract}


GAPP.NuevaÉpoca - N. ${ }^{2} 24$, noviembre2020 - ISSN: 1989-8991 - DOI:https://doi.org/10.24965/gapp.i24.10810 - [Págs. 106-119]

Incidencia de las políticas globales en las legislaciones nacionales: el caso de la Convención para la Eliminación de Todas las..

Lydia Candelaria González Orta

while CEDAW has served as an inspiration and international legitimization of gender equality laws, and contributed to the internationalization of the feminist movement, the increased complexity and bureaucracy of the periodic review has not meant further implementation of the Committee's recommendations. The article ends with a discussion on the main gaps in the Spanish Administration that hinder the effectiveness of the Convention in the advancement of women's human rights.

\section{KEYWORDS}

CEDAW; women's human rights; gender equality policies; feminist movement.

\section{SUMARIO}

1. INTRODUCCIÓN Y APROXIMACIÓN METODOLÓGICA AL OBJETO DE ESTUDIO; 2. EL CONTENIDO DE LA CEDAW: ORÍGENES Y EXPANSIÓN; 3. EL PROCEDIMIENTO DE REVISIÓN PERIÓDICA; 4. EL PROTOCOLO FACULTATIVO; 5. LA REVISIÓN PERIÓDICA ANTE LA CEDAW: ACTORES, ESTRATEGIAS Y RESULTADOS; 5.1. EL ESTADO COMO ACTOR ANTE EL COMITÉ CEDAW; 5.2. PERSPECTIVA Y ESTRATEGIAS DEL MOVIMIENTO FEMINISTA: LOS INFORMES SOMBRA; 5.3. RECOMENDACIONES DEL COMITÉ CEDAW A ESPAÑA; 6. IMPACTOS GLOCALES DE LA CEDAW; 7. RETOS PENDIENTES PARA UNA MAYOR EFICACIA DE LA CONVENCIÓN; CONCLUSIONES; REFERENCIAS BIBLIOGRÁFICAS.

\section{INTRODUCCIÓN Y APROXIMACIÓN METODOLÓGICA AL OBJETO DE ESTUDIO1}

La Convención para la Eliminación de Todas las Formas de Discriminación contra las Mujeres (la CEDAW, por sus siglas en inglés) es uno de los nueve principales Tratados Internacionales del sistema de derechos humanos de Naciones Unidas. Ha sido ratificada por prácticamente todos los países del mundo -entre ellos España en 1984-, que acceden a la revisión periódica de sus avances prevista en el Tratado, contribuyendo a la tendencia global hacia el reconocimiento de los derechos de las mujeres (Díaz y González, 2016).

Si bien el proceso de revisión periódica de los Estados ante el Comité CEDAW venía gozando cada vez de mayor reputación, el presente artículo se escribe en un momento crítico para la continuación del trabajo regular de los Comités de Tratados Internacionales de Derechos Humanos. Tras la crisis presupuestaria de 2019 que estuvo a punto de cancelar numerosas sesiones de revisión de los diferentes comités, la ONU abordará en los próximos meses la reforma profunda de los órganos de Tratados de Derechos Humanos hacia un sistema más sólido y simple. Por lo que toca a España, la publicación de las Observaciones finales correspondientes al periodo 2015-2018 está prevista en febrero de 2021 (78. ${ }^{a}$ sesión). No obstante, una reciente decisión del Comité que condena al Estado en virtud del Protocolo Facultativo por un caso de violencia obstétrica, pone de nuevo de actualidad la Convención, sus mecanismos y su efectividad. Por ello, se hace necesario realizar un balance del impacto de la Convención en las políticas de igualdad que permita valorar la relevancia de los instrumentos internacionales de derechos humanos, así como los retos pendientes.

El impacto de la CEDAW en el avance de los derechos de las mujeres a nivel nacional ha sido una de las preocupaciones de la literatura académica (Cook, 1994; Facio y Morgan, 2009; Englehart y Miller, 2014; Liebowitz y Zwingel, 2014), junto con la influencia de la Convención en el derecho internacional (Hodson, 2014; Zwingel, 2006). Este artículo parte de trabajos previos que señalan el impacto de las decisiones del Comité en las políticas públicas en España (Durán, 2011; Stoffels, 2019) y que analizan críticamente las deficiencias estructurales de la Convención con el objetivo de proponer medidas que maximicen el potencial de la CEDAW (O'Rourke, 2019).

Al objeto de describir y comprender la acción de la Convención en tanto que promotora de una tendencia y una política global ${ }^{2}$ con impactos a nivel nacional, se han empleado varios de los elementos propios del análisis de políticas públicas. En la investigación que aquí se presenta, se ha analizado el papel de los acto-

\footnotetext{
1 Agradezco los comentarios y sugerencias realizados por los revisores anónimos de la revista así como por el equipo editorial, que han ayudado a mejorar la estructura e ideas del manuscrito.

2 Para un análisis del surgimiento de nuevos actores y agendas supranacionales y la erosión de la capacidad de maniobra del Estado-nación que han dado lugar a la denominada global policy, ver Hardguindéguy, 2015.
} 
GAPP.NuevaÉpoca - N. ${ }^{2} 24$, noviembre2020 - ISSN: 1989-8991 - DOI:https://doi.org/10.24965/gapp.i24.10810 - [Págs. 106-119]

Incidencia de las políticas globales en las legislaciones nacionales: el caso de la Convención para la Eliminación de Todas las..

Lydia Candelaria González Orta

res implicados en el proceso de revisión periódica, donde destacan fundamentalmente tres: 1. las expertas que integran el Comité CEDAW; 2. el Estado-nación que busca mantener su legitimidad internacional como país respetuoso de los derechos de las mujeres; y 3 . las organizaciones feministas y de derechos humanos, como representantes de la sociedad civil y del colectivo directamente afectado. Son de especial interés, desde la perspectiva aquí empleada, la conducta e interacciones de los actores implicados, sus intereses, y los recursos que logran movilizar para influir en el procedimiento.

España es uno de los Estados Partes veteranos y regulares en el procedimiento CEDAW, lo cual permite analizar el funcionamiento del Comité y la evaluación periódica desde los ochenta. Para la realización de un análisis documental que ofreciera descripciones profundas del fenómeno a estudiar, se han seleccionado principalmente los documentos oficiales, Observaciones finales y de ONG producidos en la primera y última evaluación, obteniendo una comparación entre 2015 y 1987. Además, se han consultado diversos documentos oficiales correspondientes a las evaluaciones de 1992, 1999, 2004 y 2009, así como los primeros informes sombra presentados en $2009^{3}$.

Tanto las Observaciones finales a España como los informes sombra han aportado información relevante sobre los resultados alcanzados por el procedimiento de revisión periódica y sobre los impactos a nivel nacional de la implementación de la Convención y sus recomendaciones. El análisis de la documentación del procedimiento CEDAW ha demostrado, por tanto, ser una herramienta válida para estudiar el estado de los derechos humanos de las mujeres y las políticas de igualdad en España, así como para conocer las demandas y aspiraciones del movimiento feminista en torno a los mecanismos internacionales de derechos humanos.

Finalmente, con el objetivo de conocer de primera mano la perspectiva de los actores en este proceso, se han realizado entrevistas semiestructuradas con representantes de los tres perfiles: organizaciones feministas, informantes clave en la Administración Pública y expertas del Comité4. De esta forma, se han obtenido tres visiones diferentes sobre el proceso de revisión periódica ante la CEDAW, sobre cómo abordarlo desde España, sus fortalezas, limitaciones, e impactos a nivel nacional.

\section{EL CONTENIDO DE LA CEDAW: ORÍGENES Y EXPANSIÓN}

La CEDAW fue aprobada por la Asamblea General de Naciones Unidas en 1979 y venía a confirmar la necesidad de establecer mecanismos de control ante la violación de los derechos de las mujeres. La Convención logró salir adelante en medio de la política de la Guerra Fría gracias a un intenso trabajo diplomático de las mujeres involucradas en la Comisión sobre la Condición Jurídica y Social de las Mujeres (CSW, por sus siglas en inglés) (ver Fraser, 1995; De Haan, 2010; Hawkesworth, 2012; y Englehart y Miller, 2020). Lejos de una aproximación feminista liberal, el preámbulo de la CEDAW fue muy crítico con otros sistemas de opresión -apartheid, sionismo, colonialismo, explotación económica- en tanto que afectaban a millones de mujeres y siguiendo el eslogan all political issues are women's issues. Este potencial crítico es único entre los Tratados del sistema de derechos humanos de Naciones Unidas y ha quedado prácticamente olvidado por la literatura especializada y por las organizaciones feministas.

La Convención contiene treinta artículos que comprenden una carta internacional de derechos de las mujeres sin distinción entre derechos de tradición liberal (civiles y políticos) o de tradición socialista (económicos, sociales y culturales). La CEDAW no solo obliga a los Estados Partes a sancionar la discriminación, sino también a prevenirla, siendo de aplicación tanto en el espacio público como en el privado. La Convención abarca no solo la protección de los derechos de las mujeres frente al Estado, sino que aborda también los hechos entre particulares afirmando que los Estados deben garantizar la no discriminación en el plano «horizontal», incluso en el seno de la familia (ver art.2 y CEDAW/C/GC/32: 11). En los últimos años, el Comité CEDAW ha puesto sobre la mesa las obligaciones extraterritoriales de los Estados en materia de derechos

3 Los documentos analizados incluyen los oficiales de Naciones Unidas con signatura A/42/38; A/47/38; A/54/38/REV.1(SUP); CEDAW/C/SR.649; CEDAW/C/ESP/CO/6/Add.1; AA/follow-up/Spain/51; y CEDAW/C/ESP/CO/7-8. Además, los informes sombra presentados en 2009 por CERMI, Fundación Secretariado Gitano e Impacto de Género Ya; y en 2015 por WILPF España, Amnistía Internacional, Women's Link y la Plataforma CEDAW Sombra España. Disponibles en: https://tbinternet.ohchr.org/_layouts/15/TreatyBodyExternal/SessionsList.aspx?Treaty=CEDAW.

4 Durante abril y mayo de 2019 se realizaron en Madrid entrevistas semiestructuradas como informantes clave a Soledad Murillo, Begoña San José y la Oficina de Derechos Humanos del Ministerio de Asuntos Exteriores, a quienes agradezco su participación en la investigación. 
GAPP.NuevaÉpoca - N. ${ }^{2} 24$, noviembre2020 - ISSN: 1989-8991 - DOI:https://doi.org/10.24965/gapp.i24.10810 - [Págs. 106-119]

Incidencia de las políticas globales en las legislaciones nacionales: el caso de la Convención para la Eliminación de Todas las..

Lydia Candelaria González Orta

humanos de las mujeres, incluyendo las actividades de empresas transnacionales, a raíz de las demandas de organizaciones de derechos humanos.

Los tres principios fundamentales en los que basa su actuación el Comité CEDAW, y que aparecen explícita o implícitamente en el articulado de la Convención y la posterior interpretación del Comité en Recomendaciones Generales (en adelante, Rec. Gral.) y Observaciones finales, son: 1. igualdad sustantiva, que va más allá de la mera igualdad legal o formal, aspirando a un cambio real y tangible en el status de las mujeres; 2 . no discriminación, principio definido en el artículo 1 de la Convención y que comprende tanto la discriminación directa como la indirecta, no intencionada, de una norma o política; y 3. diligencia debida por parte de los Estados que voluntariamente aceptan las obligaciones que comporta la Convención en materia de legislación (art. 2), de políticas activas (art. 3) y de rendición de cuentas mediante los procedimientos previstos en el Tratado (Facio y Morgan, 2009).

La CEDAW, si bien aspiraba a la igualdad como proyecto transformador de la sociedad (Laporta, 2016), no mencionaba algunos temas hoy considerados parte indiscutible de los derechos humanos de las mujeres, como los derechos de las mujeres $L B T I$, el derecho al aborto o la violencia de género, siendo uno de los límites del marco internacional de derechos humanos en los setenta (De Haan, 2010: 2; Pietilä, 2007: 30). Posteriormente, Naciones Unidas aprobó la Declaración para la Eliminación de Todas las Formas de Violencia contra las Mujeres (1993) y se incluyó la violencia de género en el ámbito de protección de la CEDAW mediante dos Rec. Gral. en 1989 y 1992.

El contenido de la CEDAW se ha venido ampliando, renovando e interpretando por parte del Comité desde los ochenta a través de sus más de treinta Recomendaciones Generales sobre muy diversos temas. La Convención es un instrumento dinámico, como ha expresado el propio Comité. A partir de los noventa, con la Rec. Gral. núm. 19 y núm. 21 sobre violencia contra las mujeres y matrimonio y relaciones familiares, respectivamente, se produce un salto cualitativo en las Recomendaciones Generales del Comité, las cuales se adentran en la formulación de conceptos, definición de líneas estratégicas, y realizan una labor de divulgación y ampliación del contenido de la Convención.

La abundante producción de Recomendaciones Generales y los cambios que suponen en cuanto a cobertura de derechos en el marco de la Convención, son indicativos del grado de conciencia existente en el Comité sobre la naturaleza cambiante de los derechos humanos, las conexiones e interdependencia entre todos ellos, y la necesidad de revisión y adaptación a las circunstancias políticas y sociales. De hecho, el Comité ha sido en no pocas ocasiones pionero en la acogida y defensa de las ideas más innovadoras que, tanto desde las investigaciones feministas como desde el movimiento, se han ido introduciendo en el debate público. Algunas de ellas son las medidas especiales de carácter temporal o acción positiva (1988), la violencia contra las mujeres (1989), la necesidad de contabilizar el trabajo doméstico en el Producto Nacional Bruto (1991); o, por poner un último ejemplo, las conexiones entre el cambio climático, los desastres medioambientales y las relaciones de género, siendo la Rec. Gral. más reciente (2018) ${ }^{5}$.

El Comité ha asumido la urgencia y pertinencia de estos temas, impulsándolos desde la arena internacional y en un marco de derechos humanos. Sin embargo, existe un debate jurídico sobre la obligatoriedad de las Recomendaciones Generales, dado que no han sido ratificadas por los Estados Partes. Una de las estrategias que ha desplegado el Comité en sus Observaciones finales es la mención insistente de las Recomendaciones Generales, dándoles tratamiento de obligado seguimiento para los Estados. En España, hay juristas que defienden que las interpretaciones de los Comités de Tratados internacionales de derechos humanos ratificados por el Estado son de observancia obligada. Se apoyan en el artículo 10.2 de la Constitución y en el hecho de que España ha ratificado dicho Tratado y asumido libremente la autoridad de su Comité (Gutiérrez, 2018).

\section{EL PROCEDIMIENTO DE REVISIÓN PERIÓDICA}

El Comité CEDAW lleva más de tres décadas evaluando los derechos de las mujeres y las políticas de igualdad de género alrededor del mundo. El proceso de supervisión del cumplimiento de la Convención mediante el examen de los informes inicial y periódicos de los Estados Partes, recogido en el art.18 y similar al del resto de Tratados de derechos humanos, se ha complejizado, ampliado y procedimentado más en los

5 En el momento de finalizar este artículo, se encuentra en periodo de consulta pública con organismos de Naciones Unidas y sociedad civil la futura Rec. Gral. núm. 38 sobre tráfico de mujeres y niñas en el contexto de migración global. 
GAPP.NuevaÉpoca - N. ${ }^{2} 24$, noviembre2020 - ISSN: 1989-8991 - DOI:https://doi.org/10.24965/gapp.i24.10810 - [Págs. 106-119]

Incidencia de las políticas globales en las legislaciones nacionales: el caso de la Convención para la Eliminación de Todas las..

Lydia Candelaria González Orta

últimos años ${ }^{6}$. Además de los informes periódicos, el Comité podría solicitar a un Estado Parte un informe con carácter excepcional, así como información adicional de forma previa a la sesión.

Los dos principios fundamentales de la composición del Comité son la independencia y la representación geográfica equilibrada, en base a las propuestas realizadas por los Estados Partes. El Comité se reúne anualmente para examinar los informes presentados por un periodo no superior a dos semanas ${ }^{7}$, convocando de forma previa un grupo de trabajo reducido que elabora listas de asuntos y preguntas, e informa anualmente a la Asamblea General de sus actividades y recomendaciones realizadas.

En sus primeros años de actividad, el Comité se encontraba con numerosas dificultades debido, entre otros factores, a los retrasos y deficiencias de forma de los informes gubernamentales. De lo previsto en el Reglamento del Comité para los «casos en que los informes no se presentan o se presentan tarde» (art. 49), se deduce que una de las estrategias de resistencia a la evaluación por parte de los Estados ha consistido en el ninguneo a las normas de procedimiento con informes incompletos o ausencia de respuesta, entorpeciendo así la eficacia del proceso. Conviene señalar que las Observaciones finales del Comité vienen ofreciendo desde hace muchos años asistencia técnica a aquellos países que la necesiten por el grado de desarrollo de sus sistemas de datos. Solo unos pocos países han podido evitar la exposición de sus "trapos sucios» en materia de derechos de las mujeres: Estados Unidos, El Vaticano, Somalia, Sudán, Irán, Palaos y Tonga, países que no han ratificado la Convención, pero también países como Dominica, debido al llamativo retraso en la presentación de su informe inicial.

El Reglamento del Comité prevé que podrán participar y presentar informes en sus sesiones los organismos especializados, organismos intergubernamentales y órganos de Naciones Unidas. Pero sin duda la participación más destacada y relevante que ha marcado este proceso ha sido la de las ONG en defensa de los derechos humanos de las mujeres y/o feministas. Con la aparición de los llamados informes sombra, práctica extendida también en otros Tratados, el procedimiento de revisión periódica ante el Comité se hizo más participativo y se enriqueció con las aportaciones de informes críticos, basados en información sobre el terreno, que tienen la capacidad de influir en las decisiones del Comité.

Por tanto, la labor del Comité CEDAW consiste fundamentalmente en una evaluación de las políticas de igualdad de los Estados. Dichas políticas de ámbito nacional se ven influidas por la tendencia global que marcan los organismos internacionales y las agendas de las redes feministas transnacionales, así como por las recomendaciones del Comité. La revisión periódica a los Estados comienza a percibirse como una alternativa a las aproximaciones cuantitativas reduccionistas de un fenómeno social complejo (como los rankings e índices globales), por no mencionar los efectos perversos de dirigir la atención de los Estados hacia la mejora en unos pocos indicadores. El procedimiento CEDAW se presenta como un proceso más complejo, cualitativo y contextualizado, más acertado como herramienta de evaluación de los progresos en igualdad de género, que da cuenta de la complejidad del fenómeno y lo enmarca en el contexto específico de cada país. Asumiendo que el proceso de diálogo del Comité con los Estados Partes puede tener otras limitaciones, al menos renuncia a la sobre-simplificación de lo que significa una mejora real en la situación de las mujeres. De esta forma, se alinea con los análisis y reflexiones profundas que requiere la aproximación a la igualdad de género desde el discurso feminista y las normas internacionales de derechos humanos (Liebowitz y Zwingel, 2014).

\section{EL PROTOCOLO FACULTATIVO}

Aunque los mecanismos y uso del Protocolo Facultativo a la CEDAW no son objeto de análisis de este artículo, es necesario hacer una precisión sobre el Protocolo por dos razones: 1. los dictámenes del Comité en virtud del Protocolo son incluidos de forma sistemática en las Observaciones finales a los países como forma de seguimiento de su cumplimiento; y 2. uno de los casos de violación de derechos humanos llevados ante el Comité activando el Protocolo, el caso Ángela González Carreño c. España, ha tenido un impacto en la opinión pública española más trascendental que cualquiera de las revisiones periódicas.

6 Hasta mitad de los noventa, el Comité celebró una sesión por año, mientras que a partir de 2006 se generalizan las tres sesiones por año y en 2013 aparecen las pre-sesiones. En 2018 y 2019 ha habido seis sesiones por año, varias de ellas preparatorias, y la agenda para los próximos años apunta en la misma dirección.

7 El Comité lleva años solicitando a los Estados que apoyen la revisión del art. 20 de la Convención que restringe el tiempo del que dispone el Comité, dado su creciente volumen de trabajo. 
GAPP.NuevaÉpoca - N. ${ }^{2} 24$, noviembre2020 - ISSN: 1989-8991 - DOI:https://doi.org/10.24965/gapp.i24.10810 - [Págs. 106-119]

Incidencia de las políticas globales en las legislaciones nacionales: el caso de la Convención para la Eliminación de Todas las..

Lydia Candelaria González Orta

El Protocolo Facultativo, aprobado por la Asamblea General en 1999, suponía un salto cualitativo en la rendición de cuentas, ya que la Convención carecía de mecanismos de denuncia por violación de los derechos reconocidos en la misma. La Plataforma de Acción de Beijing, junto con la campaña que comenzaran en los noventa organizaciones feministas involucradas en los caucus y foros internacionales, supusieron un impulso al proyecto iniciado por la CSW relacionado con el derecho de petición. El Protocolo prevé que el Comité CEDAW (art.2) pueda aceptar comunicaciones individuales y colectivas contra un Estado Parte del Protocolo por violación de cualquiera de los derechos enunciados en la Convención, tras haber agotado todos los recursos de la jurisdicción interna del país. Además, el art.8 faculta al Comité para tomar la iniciativa e iniciar investigaciones sobre violaciones graves o sistemáticas de los derechos de las mujeres en un Estado Parte, tras haber recibido información fidedigna al respecto. Aunque el Protocolo no acepta reservas, sí incluye una cláusula que permite a los Estados no reconocer la competencia del Comité para realizar investigaciones en el país.

Las comunicaciones individuales tienen el potencial de denuncia de una situación específica mostrando las causas estructurales de dicha violación de los derechos de las mujeres en un país (Hodson, 2014: 570). En aquellos casos en que el Comité concluye que el Estado ha incumplido sus obligaciones en virtud de la Convención, sus dictámenes buscan el doble objetivo de solicitar una reparación del daño a la víctima (por lo general, una indemnización económica) y hacer recomendaciones de carácter general que eviten nuevos casos similares. De esta forma, el Protocolo permite una acción del Comité orientada a la solución de problemas atendiendo a sus causas y no solo a la reprobación del Estado, donde el debate social generado cobra gran importancia (Stoffels, 2019).

La combinación de ambos instrumentos, Convención y Protocolo opcional, hace que el mecanismo CEDAW sea la herramienta global más potente para la defensa de los derechos humanos de las mujeres. Mientras que la Convención exige la presentación de informes periódicos abordando la situación general en el país, el Protocolo permite el examen de casos concretos y su necesaria reparación. El Comité, a iniciativa de las organizaciones feministas y de derechos humanos, ha sido muy activo en el uso de los mecanismos que establece el Protocolo, a pesar de que una alta tasa de comunicaciones son inadmitidas por cuestiones procedimentales y de que el procedimiento de investigación ha permanecido infrautilizado (O'Rourke, 2019). Las comunicaciones individuales se han incrementado notablemente en los últimos años, lo cual indica que se ha extendido el recurso a este procedimiento, pero también que la Administración de Justicia de numerosos países continúa dando un trato y reparación deficientes a los casos de discriminación y violencia contra las mujeres.

\section{LA REVISIÓN PERIÓDICA ANTE LA CEDAW: ACTORES, ESTRATEGIAS Y RESULTADOS}

\subsection{El Estado como actor ante el Comité CEDAW}

Desde 1987, hasta en cinco ocasiones han participado diferentes gobiernos españoles en el proceso de evaluación ante la CEDAW ${ }^{8}$. La primera evaluación a España tuvo lugar en el sexto periodo de sesiones del Comité, cuando había apenas 92 Estados Partes de la Convención. Uno de los elementos clave de la participación del Estado ante la CEDAW es la formación del equipo de representantes de la delegación nacional que defenderán la gestión en materia de igualdad ante el Comité. Esa primera delegación española estuvo encabezada por Carlota Bustelo, entonces directora del Instituto de la Mujer, que aún no había recibido un mandato legal explícito de desarrollar políticas de igualdad cuando se envía el informe inicial de España (Durán, 2011: 56). Esta etapa coincidía con el origen y desarrollo de las políticas de igualdad en el país, en concreto con los cambios legislativos introducidos por Unión de Centro Democrático y la primera parte de europeización y feminismo institucional con el Partido Socialista, de 1982 a 1992 (Lombardo y León, 2015).

La primera evaluación ante la CEDAW en 1987 sirvió al Gobierno para mostrar ante instancias internacionales sus cambios legislativos en materia de discriminación contra las mujeres y primeras políticas de igualdad, así como para legitimar su gestión en línea con el discurso de los derechos humanos. El Instituto

8 La siguiente evaluación ha sufrido un retraso debido a la crisis presupuestaria de los Comités de Tratados y se prevé celebrar en la 78. ${ }^{a}$ sesión, en febrero de 2021. La pre-sesión ha tenido lugar en julio de 2019 habiéndose presentado el informe oficial del Gobierno socialista en el que se considerará buena parte del periodo de Gobierno del Partido Popular. Se han presentado también tres informes sombra. 
GAPP.NuevaÉpoca - N. ${ }^{2} 24$, noviembre2020 - ISSN: 1989-8991 - DOI:https://doi.org/10.24965/gapp.i24.10810 - [Págs. 106-119]

Incidencia de las políticas globales en las legislaciones nacionales: el caso de la Convención para la Eliminación de Todas las..

Lydia Candelaria González Orta

de la Mujer aprovechó también de forma estratégica la oportunidad de este foro internacional para dejar patente la necesidad de dotar de recursos económicos a las incipientes estructuras de igualdad (ONU, A/42/38). Las autocríticas del Gobierno se centraron en la persistencia de la segregación horizontal en la elección de estudios y el empleo, el escaso interés de las españolas por la política, la aún deficiente prestación de servicios sociales, entre otras. La delegación española recibió el reconocimiento de las expertas del Comité por su labor y compromiso, así como el enfoque crítico y la franqueza (ONU, A/42/38).

Desde aquella primera evaluación hasta 2015, tanto las políticas de igualdad de género como el movimiento feminista en España han experimentado cambios importantes, así como los roles de ambos actores, Estado y Comité. España ha pasado de una actitud de país en desarrollo en los ochenta, mostrando sus tímidos avances con orgullo ante instancias internacionales pero también sus deficiencias, haciendo incluso autocrítica en su diálogo con el Comité, a una actitud de país perteneciente a una región privilegiada del mundo como la Unión Europea, en los últimos años. España muestra ahora con sus informes y explicaciones ante el Comité su necesidad de presentarse ante instancias internacionales como un país avanzado en materia de igualdad y referente en políticas activas, como lo fue en su día con las leyes orgánicas de violencia de género (2004) y de igualdad efectiva (2007), recibiendo elogios por parte del Comité. Por su parte, a medida que aumentaban las capacidades del país en materia de políticas de igualdad, el tono del Comité en estas evaluaciones se ha endurecido, siendo más exigente, especialmente cuando se ha tratado de presentar políticas regresivas en materia de igualdad como favorables a la situación de las mujeres, como ocurriera en 2015.

Conviene recordar que España fue uno de los 16 países examinados en 2015 por la CEDAW, en un contexto marcado por la crisis económica, políticas de ajuste y conservadurismo en materia de igualdad de género, según el informe sombra presentado en Ginebra en noviembre de 2014 por la Plataforma CEDAW sombra y según organismos de la propia Naciones Unidas. Al margen del reconocimiento a los avances legislativos y adopción de tratados de derechos humanos en los últimos años, el Comité fue especialmente duro en esta revisión periódica con la gestión del Gobierno, particularmente con el impacto de género negativo de las políticas de austeridad.

Otro de los factores clave del cambio de relación entre el Estado Parte y el Comité a lo largo de estas décadas ha sido el acceso a la información. En la primera evaluación de 1987 se observa un diálogo genuino, y hasta cierto punto inocente, entre las partes, en el que el Comité hacía preguntas al Estado para las que no podía obtener datos actualizados de fácil acceso. En la actualidad, el Comité no solo dispone de información detallada y actualizada de las políticas y la situación de las mujeres en un país como España, sino que cuenta con el punto de vista crítico de los informes sombra que complementan la información oficial.

\subsection{Perspectiva y estrategias del movimiento feminista: los informes sombra}

El Comité comienza a recibir informes críticos por parte de la sociedad civil en los noventa (ver Cook, 1994: 24). Por lo que se refiere al caso español, ni el movimiento feminista ni las ONG internacionales que trabajan en España han hecho uso de este mecanismo de seguimiento de la Convención hasta el año 2009, en que se presentan ya varios informes sombra. Una de las entrevistadas lo relaciona con la escasa internacionalización del movimiento feminista español hasta hace algunos años y los débiles lazos que existían entre las organizaciones de base y las ONG de cooperación al desarrollo, más familiarizadas con estos procesos ante instancias internacionales:

Yo creo que en general el movimiento feminista en España es muy poco internacionalista y los temas de Naciones Unidas no los ha trabajado mucho, a diferencia del feminismo latinoamericano que está muy conectado a Naciones Unidas. Begoña San José, miembro de organizaciones feministas.

El punto de inflexión de la participación de organizaciones feministas en el proceso de revisión periódica ante el Comité CEDAW se produjo en 2015. Se presentaron cuatro informes sombra o alternativos, pero uno de ellos consistió en un informe conjunto presentado por más de sesenta organizaciones feministas trabajando en una plataforma creada al efecto durante más de un año. Este informe sombra conjunto fue sin duda el más completo, transversal y holístico, incluyendo información acerca de cada artículo de la Convención. Prácticamente todos los temas objeto de preocupación por el Comité habían sido incluidos en el informe sombra, como la necesidad de realizar una evaluación del impacto de género de las políticas de 
GAPP.NuevaÉpoca - N. ${ }^{2} 24$, noviembre2020 - ISSN: 1989-8991 - DOI:https://doi.org/10.24965/gapp.i24.10810 - [Págs. 106-119]

Incidencia de las políticas globales en las legislaciones nacionales: el caso de la Convención para la Eliminación de Todas las..

Lydia Candelaria González Orta

austeridad, las condiciones restrictivas para ejercer el derecho al aborto en el caso de mujeres jóvenes, o la falta de implementación del Dictamen del Comité sobre el caso González Carreño c. España (Comunicación núm. 47/2012 sobre fallos en el sistema de protección de menores en casos de violencia de género).

Además de la capacidad de influencia que logró ejercer este informe sombra en las observaciones del Comité, el proceso de elaboración generó un importante impulso a las actividades en clave global de las organizaciones feministas y el resultado generado con amplio consenso (más de 250 organizaciones firmantes) situaba sus líneas de acción como puntos de referencia para las políticas de igualdad en el país, tal como señalaron las entrevistadas:

[El informe sombra] a nosotras nos ha educado a comprender más nuestro país, analizar más cómo están las mujeres en el conjunto del país [...] Eso ha sido un enriquecimiento. Nos ha hecho entender que si hablamos a coro somos más escuchadas [...] Esto de hacer un informe conjunto yo creo que es también una pedagogía. Begoña San José, miembro de organizaciones feministas.

Yo creo que la enorme ventaja que tiene el informe sombra es que a partir de él, siempre habrá informe sombra en España. Esté quien esté [en el Gobierno]. Eso es lo maravilloso, que se ha generado un informe sombra fantástico a partir de la plataforma. Y que esa plataforma es un punto de referencia también para las políticas públicas [...] Soledad Murillo, ex-miembro del Comité CEDAW.

\subsection{Recomendaciones del Comité CEDAW a España}

Las recomendaciones que hace el Comité a España en su primera evaluación son realmente útiles para comprender la situación social de la que partía el país en cuanto al status de las mujeres en los ochenta. Dadas las incipientes políticas de igualdad en España y la escasa información, las intervenciones del Comité estaban dirigidas a conocer la situación de los derechos de las mujeres y a manifestar su preocupación en diferentes ámbitos. En cambio, a partir del examen de 2009 se observa un grado considerable de concreción en las recomendaciones que realiza el Comité, en un estilo bastante más propositivo y fiscalizador de la actuación del Gobierno. Además, se incorporan ya las secciones habituales de las que se compone este tipo de documentos en los últimos años, dotando de una mayor sistematicidad a las recomendaciones. También en 2009, el Comité introduce la interseccionalidad, al ocuparse de la situación de las niñas y mujeres gitanas en España y de las mujeres con discapacidad (CEDAW/C/ESP/CO/6/Add.1), propiciado por los informes sombra presentados por organizaciones representantes de dichos colectivos.

La evaluación en 2015 de los informes periódicos séptimo y octavo supone un cambio importante en el tono del Comité debido al contexto de políticas regresivas en materia de igualdad y políticas de ajuste como respuesta a la recesión económica en que se emiten estas recomendaciones. El diálogo constructivo y las felicitaciones al Gobierno por sus avances legislativos de años anteriores, dan paso a la expresión de preocupación en diferentes esferas, con un discurso especialmente duro en torno a cuestiones como el impacto negativo de las medidas de austeridad sobre los derechos económicos de las mujeres y los servicios públicos, la falta de aplicación por el Estado de los dictámenes emitidos por el Comité en virtud del Protocolo, y el escaso desarrollo de las medidas especiales de carácter temporal (o acción positiva). Así mismo, el Comité emitió recomendaciones en el sentido de revertir la degradación jerárquica de las estructuras de igualdad a raíz de la eliminación del Ministerio de Igualdad en 2012, en cuanto a custodia compartida en casos de violencia de género, la pérdida de acceso a la sanidad universal por parte de migrantes en situación irregular o las restricciones en el acceso al aborto para las jóvenes menores de 18 años, entre otras (CEDAW/C/ESP/ $\mathrm{CO} / 7-8)$.

Algunos temas que no formaban parte de las Observaciones finales emitidas en 1987 y que se incorporan en sucesivas evaluaciones incluyen la política económica y atención a la diversidad. Por otra parte, entre las Observaciones finales del Comité en 1987 y 2015 hay una serie de temas persistentes, de lo cual se puede deducir que no han tenido la acción eficaz esperada por la Administración a pesar de contar con mayores recursos y un entorno legislativo y social más favorable: la brecha salarial, la segregación horizontal en la educación superior y el mercado de trabajo, los estereotipos de género en la publicidad, la educación sexual, la escasez de guarderías o las medidas de acción positiva. Otros temas en materia de igualdad que el Comité ha ido incorporando en las Observaciones finales de otros países pero que no han tenido demasiada relevancia ni consistencia en las observaciones a España, se refieren a las demandas del movimiento 
GAPP.NuevaÉpoca - N. ${ }^{2} 24$, noviembre2020 - ISSN: 1989-8991 - DOI:https://doi.org/10.24965/gapp.i24.10810 - [Págs. 106-119]

Incidencia de las políticas globales en las legislaciones nacionales: el caso de la Convención para la Eliminación de Todas las..

Lydia Candelaria González Orta

feminista en torno a conciliación y corresponsabilidad, perspectiva de género en la cooperación al desarrollo, y reivindicaciones LGTBI.

Finalmente, las expertas del Comité han insistido en todas y cada una de las evaluaciones a los Estados Partes en que la difusión de las Observaciones finales es fundamental para su plena aplicación. Dado el escaso uso de las mismas por la Administración, una de las asignaturas pendientes del Gobierno es cumplir con este encargo referente a la difusión de las Observaciones finales (Durán, 2011: 61).

\section{IMPACTOS GLOCALES DE LA CEDAW}

Desde el final de la II Guerra Mundial, ha habido una expansión de los derechos de las mujeres alrededor del mundo con diferentes recorridos nacionales que se han visto influidos por una tendencia global que promocionaba, legitimaba y moldeaba estos procesos (Díaz y González, 2016). La CEDAW, que establece un estándar en dicha tendencia global, es considerada por la literatura especializada como un hito en el discurso y la agenda globales de igualdad de género (Cook, 1994), junto con las Conferencias Mundiales sobre las Mujeres de Naciones Unidas (México, 1975; Copenhague, 1980; Nairobi, 1985; Beijing, 1995). Así mismo, es ampliamente reconocido el papel de la Convención como proceso de creación de normas globales (Zwingel, 2006).

Como el resto de Estados-nación, España se ve influida por un mundo con símbolos y modelos de lo que debe ser un Estado moderno, como el reconocimiento de los derechos de las mujeres, el respeto a los derechos humanos, y también proyectos como la escolarización universal o el sistema democrático. Seguir este modelo confiere legitimidad internacional, un reconocimiento crucial para los países democráticos y aspirantes a tener cierto peso en la arena internacional (Ramírez, 2012: 5). Baste recordar cómo las violaciones de derechos de las mujeres se han empleado en la arena internacional para desprestigiar a ciertos países e incluso justificar invasiones militares (Sharp, 2005). La idea de un mundo, unas instituciones supranacionales y una opinión pública internacional que legitiman el reconocimiento de derechos humanos y los valores democráticos, da buena cuenta del fenómeno de expansión de los derechos de las mujeres alrededor del mundo desde 1945, una tendencia global a la que no ha sido ajeno nuestro país.

La participación de España en instancias internacionales y la ratificación de Tratados de Derechos Humanos formaba parte de la búsqueda de legitimidad internacional como Estado moderno y democrático tras la Transición Española. La ratificación de la CEDAW coincidía además con la adaptación de toda la normativa a la nueva Constitución y la puesta en marcha de las primeras instituciones de igualdad en el país, como el Instituto de la Mujer, creado en 1983. De esta forma, España se convirtió en uno de los países -junto con la gran mayoría de Europa occidental y la extinta Unión Soviética- más precoces en ratificar la Convención y convertirla en norma de derecho interno, solo tres años después de su entrada en vigor (1981) ${ }^{9}$. Años más tarde, España también sería precoz en la ratificación del Protocolo Facultativo, en 2001, justo el año en que entraba en vigor. Dado que el Protocolo supone un salto cualitativo en términos de exigencia a los Estados, el número de ratificaciones no es tan exitoso como el de la Convención: mientras que 187 han introducido la CEDAW en su sistema legal, 112 han hecho lo propio con el Protocolo Facultativo.

Uno de los primeros resultados de la ratificación de la CEDAW fue la aprobación de la Ley 11/1990 de reforma del Código Civil para la aplicación del principio de no discriminación por razón de sexo (Durán, 2011: 62). A partir de ese momento, la CEDAW ha servido de inspiración en materia de derechos de las mujeres, así como de legitimación internacional, de las leyes de igualdad desarrolladas por el Estado Parte, como ocurriera en otros países (ver, para el caso latinoamericano, WHRI, 2017), tal como confirma una de las entrevistadas:

Yo creo que CEDAW ha marcado una línea clarísima cuando todavía en España las políticas específicas sobre ese tema no existían. Y de hecho, cualquier persona que tenga una responsabilidad en relación con la aplicación de estas políticas, te habla de CEDAW y Beijing. Son como las dos referencias. [...] Pero CEDAW es una cuestión que rige. Porque es una Convención muy clara. Y los informes, dentro de todo lo imbricado que son los informes de Naciones Unidas, son también bastante claros. Representante de la Oficina de Derechos Humanos del MAEC.

9 Los Estados pueden realizar reservas al articulado de la Convención, siempre que no atenten contra el objetivo de la misma Este ha sido uno de los grandes temas en la literatura, pues numerosos Estados han hecho uso y abuso de las reservas a la CEDAW. En el caso de España, se mantiene la reserva al artículo 7 sobre el derecho de las mujeres a la participación política en igualdad con los hombres, puesto que en la Constitución persiste la prevalencia del varón a la hora de acceder al trono y jefatura del Estado. 
GAPP.NuevaÉpoca - N. ${ }^{024}$, noviembre2020 - ISSN: 1989-8991 - DOI:https://doi.org/10.24965/gapp.i24.10810 - [Págs. 106-119]

Incidencia de las políticas globales en las legislaciones nacionales: el caso de la Convención para la Eliminación de Todas las...

Lydia Candelaria González Orta

Ya en 2004, la exposición de motivos de la Ley Orgánica 1/2004 de medidas de protección integral contra la violencia de género hacía expresa referencia a la Convención y su recomendación de proporcionar una respuesta global a la violencia contra las mujeres. Unos años más tarde, la Ley Orgánica 3/2007 para la Igualdad Efectiva entre mujeres y hombres destacaba la Convención como el principal texto internacional de derechos humanos que reconoce la igualdad entre hombres y mujeres como principio jurídico universal. Las Comunidades Autónomas se han hecho eco también de la legitimidad de la CEDAW, que aparece mencionada en las leyes autonómicas de igualdad y/o contra la violencia de género de Andalucía, Cataluña, Murcia, Baleares, Canarias, Castilla-La Mancha y Navarra. Por parte de las instituciones estatales, desde 2006 los sucesivos Ministerios de Asuntos Exteriores vienen citando la Convención en diversos marcos de asociación estratégica, así como en el II Plan Nacional de Acción de Mujeres, Paz y Seguridad, en el que también se alude a las Recomendaciones Generales del Comité. La Convención ha servido también para justificar las medidas de acción positiva que recoge su art.4, en aquellos ámbitos en los que se han adoptado (Durán, 2011: 64).

Por lo que se refiere al impacto de la revisión periódica, la propia naturaleza de las recomendaciones del Comité en sus primeras décadas de trabajo hace complicado un seguimiento de sus resultados, dado que carecían de la concreción suficiente. En cambio, las Observaciones finales más recientes permiten un mayor seguimiento tanto por la sociedad civil como por el propio Comité. No obstante, su impacto ha sido limitado en cuanto a aplicación directa de las recomendaciones, ya que varias de ellas se vienen repitiendo desde los ochenta, y en cuanto a repercusión social, dado que la Convención empieza a conocerse por los medios, la Administración y las organizaciones feministas solo en los últimos años. Durante mucho tiempo, la visibilidad de la CEDAW ha sido escasa a pesar de las reformas legales que había inspirado y/o legitimado (Durán, 2011). Sin embargo, el caso de España es un buen ejemplo de cómo alrededor de la CEDAW se dan impactos glocales. De lo global a lo local, la Convención ha dotado de legitimidad a la legislación en igualdad de género y violencia contra las mujeres. Ahora bien, las recomendaciones y dictámenes del Comité han tenido un alcance limitado, entre otros motivos, porque en los diferentes niveles del Gobierno y la Administración de Justicia no hay un consenso sobre la obligatoriedad de las mismas para el Estado Parte:

Es complicadísimo lo de hacer cumplir las recomendaciones. Siempre nos encontramos con lo mismo de «cómo hace usted para...» Es muy difícil porque al final no son jurídicamente vinculantes. No es una sentencia de un tribunal. Ahora bien, si lo fueran, a lo mejor tampoco tendrían la adhesión que tienen. Representante de la Oficina de Derechos Humanos del MAEC.

Quizá un resultado más directo de la revisión periódica sea el que involucra al movimiento feminista en España con la creación de una Plataforma de organizaciones que comienza a presentar informes conjuntos y mantiene su actividad hasta la siguiente revisión periódica. De lo local a lo global, el informe sombra conjunto de 2014 tuvo un impacto decisivo en las Observaciones finales del Comité, que adoptó su discurso crítico sobre los derechos económicos de las mujeres ante las políticas de ajuste.

En el ámbito judicial, la CEDAW ha tenido una mayor incidencia en resoluciones judiciales a partir de 2016, donde tanto sentencias como autos de tribunales autonómicos y estatales han invocado la CEDAW para justificar sus deliberaciones en casos de discriminación laboral, acoso sexual, violencia de género, conciliación de la vida familiar y laboral y reconocimiento de la condición de refugiada. El hecho de que no solo el articulado de la Convención, sino también las Recomendaciones Generales del Comité (ver por ejemplo, la Sentencia núm. 2251/2014), comiencen a ser invocadas por los Tribunales en aras de una interpretación de las leyes y los casos judiciales con perspectiva de género y acorde a los compromisos internacionales del Estado, otorga una legitimidad importante a unas Recomendaciones Generales que no son jurídicamente vinculantes ni de directa aplicación en los Estados Partes. No obstante, a pesar de su mayor incidencia en los últimos años, la Convención ha estado infrautilizada por los tribunales españoles durante décadas, ya que se ha mencionado en apenas una treintena de sentencias y autos desde su ratificación en 1984.

Pero es seguramente el uso del Protocolo Facultativo el que ha otorgado una mayor visibilidad a la CEDAW en nuestro país, en un camino abierto por el caso González Carreño c. España relativo al asesinato de una menor por su padre maltratador. En 2014, tras agotar las vías nacionales, el caso es llevado ante el Comité CEDAW en virtud del Protocolo. El Dictamen 47/2012 del Comité consideró que el Estado no había guardado la diligencia debida, uno de los principios de la Convención, para proteger a la autora y a su hija. Además de recomendar una debida indemnización a la autora, el Comité realizó recomendaciones de carácter general de forma que se puedan evitar casos similares. Precisamente un punto de inflexión importante en el papel que jugará la CEDAW en los próximos años lo ha marcado la Sentencia 1263/2018 del Tribunal 
GAPP.NuevaÉpoca - N. ${ }^{2} 24$, noviembre2020 - ISSN: 1989-8991 - DOI:https://doi.org/10.24965/gapp.i24.10810 - [Págs. 106-119]

Incidencia de las políticas globales en las legislaciones nacionales: el caso de la Convención para la Eliminación de Todas las..

Lydia Candelaria González Orta

Supremo sobre el caso. La Sentencia considera que aunque no exista en el ordenamiento jurídico español un procedimiento que posibilite la eficacia ejecutiva de las decisiones del Dictamen 47/2012, la Administración había vulnerado derechos fundamentales de la recurrente y un Dictamen como el de la CEDAW puede habilitar para formular una reclamación de responsabilidad patrimonial del Estado. El Supremo rompe así con la anterior doctrina al aceptar la relevancia en el derecho interno de los compromisos internacionales asumidos por el Estado en materia de derechos humanos (ver Gutiérrez, 2018; y Stoffels, 2019).

El segundo Dictamen de condena al Estado por su falta de diligencia debida en la aplicación de los derechos reconocidos en la Convención se refiere a un caso de violencia obstétrica (comunicación núm. 138/2018). La reciente publicación de esta Decisión del Comité, en febrero de 2020, hace que no se pueda valorar aún la difusión realizada por la Administración ni su impacto mediático. El Gobierno está emplazado a dar una respuesta por escrito ante el Comité con información sobre las medidas adoptadas en materia de reparación a la autora y de prevención de la violencia obstétrica.

\section{RETOS PENDIENTES PARA UNA MAYOR EFICACIA DE LA CONVENCIÓN}

El análisis de un proceso como el de la revisión periódica ante la CEDAW tiene el doble objetivo de comprender el procedimiento en el que intervienen diferentes actores con diversos intereses y recursos, así como evaluar sus resultados e impactos para proponer recomendaciones de mejora. El mecanismo de la Convención se basa en un diálogo constructivo con los Estados Partes, como se ha explicado anteriormente. Su eficacia e impacto pueden valorarse solo a largo plazo. Sin embargo, los impactos glocales que ha tenido la CEDAW en España no dejan de verse reflejados sobre todo en el plano declarativo, como inspiración de leyes y sentencias, insuficiente para tres décadas. En la Administración Pública española existe también una serie de retos pendientes que obstaculizan la eficacia de la Convención en el avance de los derechos de las mujeres.

En primer lugar, la ausencia de una estrategia de comunicación y difusión que otorgue una mayor visibilidad a la CEDAW en todos los niveles de la Administración. El propio Comité viene recomendando desde hace años de manera sistemática a todos los Estados Partes que den amplia difusión a sus Observaciones finales, con el doble objetivo de que las obligaciones del Estado en materia de derechos humanos sean de obligado conocimiento en la administración estatal, local y de justicia, y de que las mujeres conozcan los derechos reconocidos en la Convención. En el ámbito judicial, el Comité CEDAW viene recomendando además desde hace años una mayor formación de sus profesionales en materia de derechos humanos de las mujeres para poder interpretar los casos de discriminación por razón de género a la luz de la Convención, así como una mayor difusión de los derechos reconocidos en la CEDAW para que puedan ser invocados ante los Tribunales.

En segundo lugar, el contenido y proceso de elaboración de los informes gubernamentales se ha basado en la recopilación de acciones relacionadas con igualdad en los diferentes departamentos ministeriales, de forma que se reporte el mayor número de acciones ante Naciones Unidas. En dicho proceso no solo es limitada la concepción de la rendición de cuentas, sino también la de participación de la sociedad civil, que consiste en la recogida de comentarios y propuestas al informe oficial. En este sentido, resulta más relevante y útil la promoción de la participación del movimiento feminista y de las organizaciones de derechos humanos en el proceso de revisión periódica, mediante la presentación de informes conjuntos y la participación en las sesiones del Comité, así como en el seguimiento permanente de las Observaciones finales. El proceso de revisión periódica en numerosos países ha mostrado que en aquellos casos en que el movimiento feminista de base se involucra en la producción de informes independientes y alternativos que aportan una visión crítica y de primera mano a las expertas del Comité, las recomendaciones del Comité son más acertadas, más críticas y holísticas.

Precisamente, la ausencia de estructuras nacionales permanentes para el seguimiento de las recomendaciones emitidas por el Comité CEDAW es uno de los retos pendientes de la Administración. La necesidad de este tipo de mecanismos se ha puesto de manifiesto por diferentes organizaciones de derechos humanos participantes en el proceso de revisión periódica como una carencia de los Estados que impide la adecuada rendición de cuentas y menoscaba la eficacia de la Convención. Dicho mecanismo nacional para el seguimiento de las Observaciones finales del Comité y la implementación de sus dictámenes en virtud del Protocolo, tendría competencias en la implementación, asignación presupuestaria, diseño de indicadores y evaluación de las medidas. Un mecanismo de estas características requeriría además, según las propias 
GAPP.NuevaÉpoca - N. ${ }^{2} 24$, noviembre2020 - ISSN: 1989-8991 - DOI:https://doi.org/10.24965/gapp.i24.10810 - [Págs. 106-119]

Incidencia de las políticas globales en las legislaciones nacionales: el caso de la Convención para la Eliminación de Todas las..

Lydia Candelaria González Orta

recomendaciones del Comité, la participación de organizaciones de mujeres, feministas y de derechos humanos, garantizando uno de los principios básicos de las políticas de igualdad con enfoque de derechos humanos como es la participación de la sociedad civil (WHRI, 2017).

El tercero de los retos pendientes por parte de España alude al ámbito judicial y, en concreto, al camino abierto por la Sentencia 1263/2018 del Tribunal Supremo, que reconoce como vinculantes para el Estado las decisiones provenientes de un Comité de Tratados de Derechos Humanos ratificados por España. Plantea, no obstante, cierta incertidumbre en términos de creación de jurisprudencia de forma que se siente un precedente y se de solución análoga a dictámenes similares, otorgando con ello al Comité de poderes cuasi-judiciales en el caso del Protocolo, y al texto de la Convención y sus Recomendaciones Generales, el carácter de fuente de interpretación.

Y finalmente, la infrautilización en el diseño de las políticas de igualdad del enfoque de derechos humanos que plantean organismos de reconocido prestigio como el Comité CEDAW y el UNGDAW (Grupo de Trabajo de Naciones Unidas sobre discriminación de las mujeres en la legislación y la práctica), supone un reto en el contenido y alcance de las políticas. Las nuevas demandas relacionadas con la diversidad sexual y de identidad de género, las críticas al modelo económico y su crisis climática, o la gestión de desastres y emergencias sanitarias con perspectiva de género, no solo han tenido un desarrollo en las Recomendaciones Generales del Comité, sino que se vienen incorporando en las Observaciones finales más recientes. Sin embargo, dichas recomendaciones innovadoras tienen un escaso recorrido en la implementación a nivel nacional, y las Recomendaciones Generales están infrautilizadas por diversas razones, entre las que se encuentra su desconocimiento por los Estados y las propias organizaciones feministas y su consideración como adendas o interpretaciones del Comité sin vinculación jurídica.

\section{CONCLUSIONES}

Este artículo ha comenzado con la descripción de los mecanismos que prevé la CEDAW para analizar el papel de los diferentes actores clave en el caso español y la evolución experimentada desde los ochenta. Durante los primeros exámenes ante el Comité (1984 a 2004), el feminismo institucional tuvo el protagonismo en este proceso, dado su papel en el impulso de las políticas de igualdad de género en España, mientras que en los últimos años (y especialmente en el último examen de 2015), ha habido un resurgimiento de la movilización feminista en torno a la CEDAW y otros espacios de Naciones Unidas, como Beijing+20. Por tanto, se observa una evolución desde una participación más institucional y formal, a una participación y protagonismo compartidos con las organizaciones feministas y de derechos humanos.

Se han abordado a continuación los impactos glocales de la CEDAW desde un planteamiento teórico sobre la participación de los Estados en instancias internacionales y su seguimiento de tendencias globales como parte de la búsqueda de legitimidad internacional de los Estados modernos, puesto que la CEDAW y sus mecanismos forman parte de esta tendencia. En el caso español, la participación como Estado Parte en la CEDAW coincidía con la Transición Española, la puesta en marcha de las primeras instituciones de igualdad en el país y la adaptación de toda su normativa a la nueva Constitución.

La CEDAW ha tenido tres impactos principales en este tiempo en nuestro país: ha servido de inspiración en materia de derechos de las mujeres, así como de legitimación internacional de las leyes de igualdad desarrolladas por el Estado, y ha contribuido a la articulación y proyección internacionales del movimiento feminista. Sin embargo, como se ha argumentado a lo largo del artículo, la mayor complejidad y burocratización del proceso de revisión periódica no se ha traducido en una mejor y directa aplicación de las recomendaciones del Comité y de sus decisiones emitidas en virtud del Protocolo Facultativo.

La CEDAW se basa fundamentalmente en recomendaciones y en un diálogo constructivo con el Estado Parte (Liebowitz y Zwingel, 2014). Dicho en términos sociológicos, la tendencia global hacia el reconocimiento de derechos de las mujeres se expande mediante influencias que moldean las identidades y no con instrumentos de coerción o imposición (Ramírez, 2012: 3). No obstante, en todos los Estados existe también una serie de retos pendientes que obstaculizan la eficacia de la Convención en el avance de los derechos humanos de las mujeres. En esto España no es una excepción.

El examen periódico -o si se prefiere, diálogo constructivo- de la CEDAW es un proceso con unas claras reglas del juego en el que cada actor tiene su papel. Los Estados Partes fijan su posición e imagen internacional como defensores del principio de igualdad de género a través de los documentos oficiales que someten a evaluación y de las delegaciones gubernamentales de alto nivel que envían a las sesiones 
GAPP.NuevaÉpoca - N. ${ }^{2} 24$, noviembre2020 - ISSN: 1989-8991 - DOI:https://doi.org/10.24965/gapp.i24.10810 - [Págs. 106-119] Incidencia de las políticas globales en las legislaciones nacionales: el caso de la Convención para la Eliminación de Todas las..

Lydia Candelaria González Orta

presenciales con el Comité. Por su parte, el Comité cuenta como recurso fundamental con la legitimidad del sistema de Derechos Humanos de Naciones Unidas en su actividad y recomendaciones, que se plasman en los documentos analizados en este artículo. La voz de las organizaciones feministas y de derechos humanos, plasmada en informes sombra y campañas de comunicación, se encuentra en desventaja frente a los otros dos actores y es también diversa y desigual dentro de las redes feministas transnacionales.

Tanto el Comité como las organizaciones feministas y de derechos humanos usan como recurso la capacidad de influir en una opinión pública internacional cada vez más sensible a las violaciones de derechos humanos de las mujeres. Hasta el momento, y a pesar de las deficiencias estructurales y la crisis del sistema de Tratados, la combinación de los procedimientos previstos en la CEDAW y su Protocolo constituye la mejor herramienta global para hacer efectivos los derechos humanos de las mujeres.

\section{REFERENCIAS BIBLIOGRÁFICAS}

ASAMBLEA GENERAL DE NACIONES UNIDAS (1987): Informe del comité para la eliminación de la discriminación contra la mujer (sexto período de sesiones) suplemento núm. 38. A/42/38. URL: https://www.undocs.org/A/42/38.

COMITÉ CEDAW (2011): Observaciones finales del Comité para la Eliminación de la Discriminación contra la Mujer: España. CEDAW/C/ESP/CO/6/Add.1. URL: https://tbinternet.ohchr.org/_layouts/15/treatybodyexternal/Download. aspx?symbolno=CEDAW\%2FC\%2FESP\%2FCO\%2F6\%2FAdd.1\&Lang=en.

COMITÉ CEDAW (2014): Recomendación general núm. 32 sobre las dimensiones de género del estatuto de refugiada, el asilo, la nacionalidad y la apatridia de las mujeres. CEDAW/C/GC/32. URL: https://undocs.org/ CEDAW/C/GC/32.

COMITÉ CEDAW (2015): Observaciones finales sobre los informes periódicos séptimo y octavo combinados de España. CEDAW/C/ESP/CO/7-8. URL: https://tbinternet.ohchr.org/_layouts/15/treatybodyexternal/Download. aspx? symbolno=CEDAW/C/ESP/CO/7-8\%20\&Lang=Sp.

COOK, R. (1994): Human Rights of Women: National and International Perspectives. Philadelphia, PA, USA: University of Pennsylvania Press.

DE HAAN, F. (2010): “Continuing Cold War Paradigms in Western Historiography of Transnational Women's Organizations: the case of the Women's International Democratic Federation (WIDF)", en Women's History Review, vol. 19, núm. 4, págs. 547-573. Fecha de consulta: 14-06-2020. DOI: https://doi.org/10.1080/09612025.2 010.502399.

DÍAZ, C. ; GONZÁLEZ, L. (2016): "Revueltas árabes y movimientos feministas transnacionales en la sociedad global”, en Revista Española de Sociología, vol. 25, núm. 1, págs. 89-108. URL: https://recyt.fecyt.es/index.php/res/article/ view/65407.

DURÁN Y LALAGUNA, P. (2011): "The CEDAW's reception in Spain: the gap between law and practice", en Yearbook of humanitarian action and human rights, núm. 9, págs. 53-66. DOI: http://dx.doi.org/10.18543/aahdh-02011pp53-66.

ENGLEHART, N.; MILLER, M. (2020): "Women's Rights in International Law: Critical Actors, Structuration, and the Institutionalization of Norms", en Politics \& Gender, vol. 16, núm. 2, págs. 363-387. DOI: https://doi.org/10.1017/ S1743923X19000242.

ENGLEHART, N.; MILLER, M. (2014): “The CEDAW Effect: International Law's Impact on Women's Rights”, en Journal of Human Rights, vol. 13, núm. 1, págs. 22-47. DOI: https://doi.org/10.1080/14754835.2013.824274.

FACIO, A.; MORGAN, M. (2009): "Equity or Equality for Women? Understanding CEDAW's Equality Principles", en IWRAW Occasional Paper, núm. 14. DOI: http://dx.doi.org/10.2139/ssrn.1469999.

FRASER, A. (1995): "The Convention on the Elimination of all Forms of Discrimination against Women (The Women's Convention)", en WINSLOW, A. (ed.): Women, Politics and the United Nations, págs. 77-94. London: Greenwood Press. 1. ${ }^{\mathrm{a}}$ ed.

GUTIÉRREZ ESPADA, C. (2018): "La aplicación en España de los Dictámenes de Comités Internacionales: La STS 1263/2018, un importante punto de inflexión", en Cuadernos de Derecho Transnacional, vol. 10, núm. 2, págs. 836-851. DOI: $h$ ttps://doi.org/10.20318/cdt.2018.4406.

HARDGUINDÉGUY, J. B. (2015): Análisis de políticas públicas. Madrid: Tecnos. 2. a ed.

HAWKESWORTH, M. (2012): Political Worlds of Women. Activism, Advocacy, and Governance in the Twenty-First Century. Boulder: Westview Press. 1. ${ }^{a}$ ed.

HODSON, L. (2014): "Women's rights and the periphery: CEDAW's optional protocol”, en The European Journal of International Law, vol. 25, núm. 2, págs. 561-578. DOI: https://doi.org/10.1093/ejil/chu027.

LAPORTA HERNÁNDEZ, E. (2016): "Desde la Convención sobre la Eliminación de todas las Formas de Discriminación de la Mujer a la igualdad transformativa en España”, en LA BARBERA, M. y CRUELLS, M. (coord.): Igualdad de género y no discriminación en España: evolución, problemas y perspectivas, págs. 59-82. Madrid: Centro de Estudios Políticos y Constitucionales.

LIEBOWITZ, D.; ZWINGEL, S. (2014): "Gender Equality Oversimplified: Using CEDAW to Counter the Measurement 
GAPP.NuevaÉpoca - N. ${ }^{2} 24$, noviembre2020 - ISSN: 1989-8991 - DOI:https://doi.org/10.24965/gapp.i24.10810 - [Págs. 106-119] Incidencia de las políticas globales en las legislaciones nacionales: el caso de la Convención para la Eliminación de Todas las...

Lydia Candelaria González Orta

Obsession”, en International Studies Review, vol. 16, núm. 3, págs. 362-389. DOI: https://doi.org/10.1111/ misr.12139.

LOMBARDO, E.; LEÓN, M. (2015): "Políticas de igualdad de género y sociales en España: Origen, desarrollo y desmantelamiento en un contexto de crisis económica”, en Investigaciones Feministas, vol. 5, págs. 13-35. DOI: https://doi.org/10.5209/rev_INFE.2014.v5.47986.

O'ROURKE, C. (2019): "Bridging the Enforcement Gap? Evaluating the Inquiry Procedure of the CEDAW Optional Protocol", en American University Journal of Gender, Social Policy \& the Law, vol. 7, núm. 1. URL: https:// digitalcommons.wcl.american.edu/jgspl/vol27/iss1/1.

PIETILÄ, H. (2007): The Unfinished Story of Women and the United Nations. New York: UN Non-Governmental Liaison Service.

RAMÍREZ, F. (2012): "The world society perspective: concepts, assumptions, and strategies", en Comparative Education, vol. 48, núm. 4, págs. 423-439. DOI: https://doi.org/10.1080/03050068.2012.693374.

SHARP, J. (2005): "Guerra contra el terror y geopolítica feminista”, en Tabula Rasa, núm. 3, págs. 29-46. Fecha de consulta: 14-06-2020. URL: https://www.revistatabularasa.org/numero03/.

STOFFELS, R. A. (2019): "The role of the CEDAW Committee in the implementation of public policies on gender issues: analysis through a study of the protection of girls' rights in Spain”, en The International Journal of Human Rights, vol. 23, núm. 8, págs. 1.317-1.336. DOI: https://doi.org/10.1080/13642987.2019.1603144.

TRIBUNAL SUPERIOR DE JUSTICA DE LAS PALMAS DE GRAN CANARIA (2014): Sentencia núm. 2251/2014 del Tribunal Superior de Justica de Las Palmas de Gran Canaria, de 22 de diciembre de 2014. STSJ ICAN 4810/2014.

TRIBUNAL SUPREMO (2018): Sentencia núm. 1263/2018 del Tribunal Supremo. Sala de lo ContenciosoAdministrativo, de 17 de julio de 2018. R. CASACION/1002/2017.

WOMEN'S HUMAN RIGHTS EDUCATION INSTITUTE (2017): «Good laws» translated into «good practices»: A Global Research Study. Latina America and the Caribbean region. University of Toronto. Fecha de consulta: 1710-2019. URL: https://www.ohchr.org/EN/Issues/Women/WGWomen/Pages/CompendiumGoodPractices.aspx.

ZWINGEL, S. (2006): "From intergovernmental negotiations to (sub)national change. A transnational perspective on the impact of CEDAW", en International Feminist Journal of Politics, vol. 7, núm. 3, págs. 400-424. DOI: https://doi. org/10.1080/1461674050016118. 